\title{
Does memory help optimise fruit fly foraging at the landscape level?
}

\author{
$\underline{\text { Jaye D Newman }}^{\text {ab }}$, Anthony R Clarke ${ }^{a}$, Rehan Silva ${ }^{a}$ and Hazel Parry ${ }^{b}$ \\ ${ }^{a}$ School of Biology and Environmental Science, Queensland University of Technology, Brisbane, QLD 4001, \\ Australia \\ ${ }^{b}$ CSIRO, GPO Box 2583, Brisbane, QLD 4001, Australia \\ Email: jaye.newman@qut.edu.au
}

\begin{abstract}
Remembering a good quality diet or patch generally has positive implications for fitness for animals, though there may be circumstances where memory could become detrimental. In the polyphagous pest insect Bactrocera tryoni (Froggatt) recent research indicates that memory contributes to their foraging strategy. When presented with a good quality host in which to lay eggs they will remember that host for a longer time than a poor host. Though when presented a poor host, the preference the following day will be for the poor host even if the good host is present, but this memory will decay quickly if they do not receive continual exposure. We wanted to know how optimal foraging strategy with and without memory would influence population and time spent in three host quality types: good, average, and poor in a local heterogeneous landscape context. An individual-based population model was developed to examine how these behaviours respond in four heterogeneous landscapes with host proportions of $30 \%$ and $60 \%$, fragmented or aggregated. There were also three fruiting scenarios: simultaneous, or sequential with good host type fruiting first, or poor host type fruiting first. The mean daily population was similar between the different landscapes, except when fruit was available simultaneously and fragmented with $60 \%$ proportion of the landscape hosts where optimal foraging fly population was much higher than fly agents with memory. When fruits were available simultaneously the fly agents that had memory spent more time in poor and average hosts than the fly agents with optimal foraging behaviour. When hosts were fruiting sequentially there was little difference between the time spent in each host type. This is an interesting first look into incorporating memory mechanisms into an individual-based model using data from a polyphagous pest.
\end{abstract}

Keywords: Memory decay, ecological modelling, agent-based, foraging 


\section{INTRODUCTION}

Individual-based modelling (IBM), also known as agent-based modelling, can incorporate processes of foraging such as memory and experience to determine emergent patterns such as population dynamics and distribution. This has been represented in IBM's in animals where memory generally has a positive influence on foraging success (Bracis et al., 2015; Gautestad, 2011). Menzel (1999) argues that memory is constrained by species specific requirements of the animal in it's natural environment and that behaviour is determined by information gathered over time and also with evolutionary selection (phylogenetic history). Research into insect experience and memory has largely been focused on the hymenopteran order in the insects and therefore the majority of IBM's that include insect memory belong this group (Becher et al, 2016; Dubois et al, 2021). Whereas in the generalist herbivores, where an evolved response to host quality choice is more likely than an instant 'reward' (Silva et al. 2020) there are few examples where memory have been incorporated into IBM's (Potting et al, 2005; Guttal et al, 2012).For generalist herbivorous insects, who discriminate between a broad range of hosts with varying qualities within and between hosts, it is hypothesised that they are at a disadvantage as the ability to remember such a broad host range is neurologically challenging (Bernays, 2001). Though a recent hypothesis "the sequential cues hypothesis" by Silva and Clarke (2020b) puts forward a potential solution to the difficulty of a broad diet breadth. They argue that generalist herbivorous insects recognise common plant cues and once in that location can then associate specialised cues (Silva and Clarke, 2020b). Research on the ovipositional foraging choice of Queensland fruit fly, Bactrocera tryoni (Froggatt), provides an interesting insight into how experience influences host choice.

Bactrocera tryoni is an Australian polyphagous pest fruit fly which infests a wide range of fruits and vegetables. The larvae are restricted to the fruit that the females oviposit into. Females have an innate ability to rank hosts in which to oviposit, and this is correlated to larval survival (Balagawi et al., 2005; Silva et al., 2020). Recently it has been shown that prior oviposition experience modifies host choice (Silva and Clarke, 2020a; Silva et al., 2020). If a female experiences oviposition into a poor host, then the following day, she is more likely to lay eggs in that poor host even if there are good quality hosts available nearby. This foraging behaviour may have implications on oviposition choice, and hence population dynamics, in a heterogenous landscape. Potentially leading to the flies getting "stuck" in a poor host as they prefer hosts which they have experienced: a "Ground hog day" scenario.

This leads us to three hypotheses: 1) That memory influences host use in a heterogeneous landscape due to the fly agents affiliating to poor hosts and hence a lower population. 2) Simultaneous fruiting scenarios will be disadvantageous to agents with memory foraging as compared to optimal foraging. 3) We expect that with aggregation, as opposed to a fragmented distribution of host types, in a landscape there will be less opportunity for a fly agent to find a better host, presenting a "Ground hog day" pattern where fly agents get "stuck" in the aggregated poor host.

\section{METHODOLOGY}

\subsection{Agent-based model of fruit fly movement behaviour}

An agent-based population dynamic model was developed using the GAMA version 1.8.1 platform (Taillandier et al., 2019). This model included larval host quality and its impact on the female adult's survival and fecundity using developmental data when B. tryoni were reared on various natural fruits (Balagawi, 2007). We simplified this data to three general host quality types: good, average, and poor. These host types are represented as a cell in the landscape grid. Fly agents move once within a daily time step within the limits of their sensing or searching boundary which are fixed at 10 and 20 meters in diameter respectively. These distances assume that females of $B$. tryoni have similar perception as males to attractants in the field (Stringer et al, 2017), and flight orientation of female Rhagoletis pomella towards virtual objects (Kaushik et al, 2020). If there are no host types fruiting within the sensing boundary then the fly agent will move to a random location within the searching boundary. The host type that the agent fly chooses determines her offspring's survival and subsequent adult fitness. Here, we focus only on the foraging behaviour rules of the female adult fruit fly agents in the model and explore two behavioural strategies: optimal foraging behaviour and foraging with memory of oviposition.

1) Optimal foraging behaviour assumes that flies have an innate ability to recognise host quality: the fly will select the best quality fruiting host type within the sensing boundary and move to this during the time step.

2) Memory of oviposition experience is based on the work of Silva et al. (2020). This research identified two main processes that occur when a fruit fly experiences various hosts in which it oviposits. One, 
the flies prefer fruit types in which they have previously laid eggs into. And two, the preference for that fruit decreases over time with flies remembering good hosts longer than poor hosts. This behaviour was incorporated into the model by using a matrix of the results of the probability of preference for the different hosts. The host quality ranking was determined by the percent survival of the offspring in (Silva et al., 2020). The rules of this behaviour are outlined in fig. 1. If an agent fly experienced a host, i.e., it chose that cell on the previous time step, then it is affiliated to that fruit and has an increased probability to move to that host quality in the next time step. If the agent fly moved away from the host type it was affiliated with then the probability of it returning to that host type decreases depending on the number of days of not reinstating the memory. If the fly agent lands on a different host type, even if it is already affiliated with another host, then it affiliates with the new host type. If the agent fly stayed with the affiliated host type, then its probability to remember this host is reset to the highest probability as shown in fig. 1.

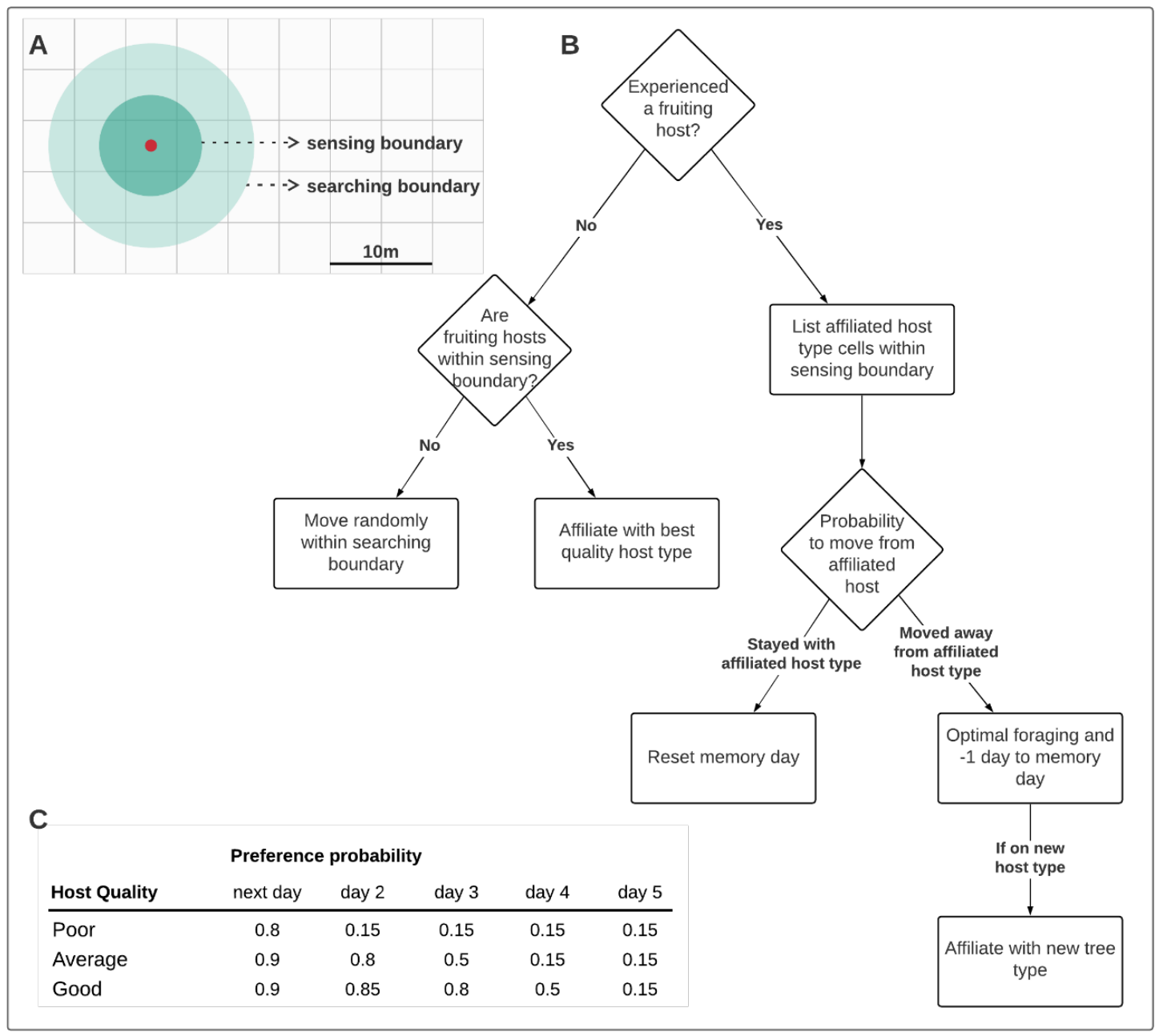

Figure 1. Description of the rules for the memory process. A) The sensing and searching boundary of a female fly agent. The sensing boundary is fixed at 10 meters and the searching radius at 20 meters. A fly agent will move once withing these boundaries at each time step. B) Flow diagram describing the sequence

of how a fly agent decides where to move during the time step. C) The matrix used to determine the probability to continue affiliation with host or choose a different host type in which to affiliate to.

\subsection{Landscape and fruiting scenarios}

Four landscapes were generated using a modified version of the Schelling segregation model in a $10 \times 10$ grid where each cell is $5 \times 5$ meters, a total of $50 \times 50$ meter grid in a torus landscape. Each cell represents a medium to large fruit tree and as we are investigating local foraging this landscape size was sufficient. The four landscape properties were $30 \%$ or $60 \%$ host proportion with the host types fully aggregated or fragmented (fig. 2). There were two fruiting scenarios, sequential and simultaneous. Simultaneous fruiting occurred three times 
in a year, whereas sequential fruiting was a single host type was fruiting at a time once each per year. Simulations of the order of fruiting were also performed for the sequential scenario for each of the landscapes with poor host fruiting first and good host fruiting first, to determine if fruiting order had an influence on emergent patterns of time spent in each host.

The model was initialised with 5 mature female flies randomly located on one of the three host types in the landscape. For the first timestep only they will move to the best fruiting host within their sensing boundary (i.e. optimal foraging) regardless of their behaviour strategy. If there are no fruiting hosts within the sensing boundary, they will move to a random location within their searching boundary. Each simulation is run for five years, and the simulation is replicated five times for each landscape type and foraging strategy. The output of cumulative days that all agent flies spent in each of the host type is recorded. The value at the last timestep, is used to calculate the percentage of time spent in each host and the average daily flies. The boxplots represent the minimum, mean, standard deviation, and maximum values. Analysis on the time spent in each host type was performed using R (RCore, 2020) and RStudio (RStudio, 2016).

A

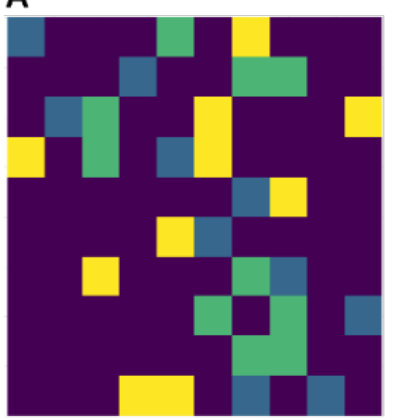

C

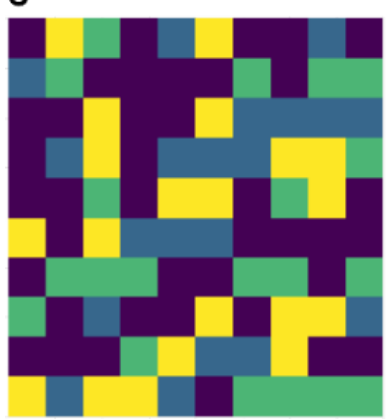

B

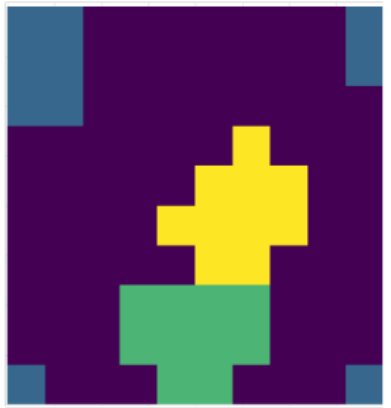

D

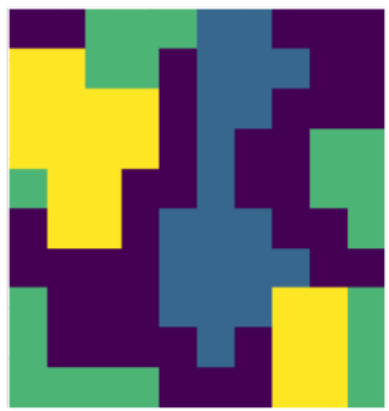

\section{Host type}

Good host

Average host

Poor host

Non host

Figure 2. Four generated landscapes. A) 30\% proportion of host types with a fragmented distribution. B) $30 \%$ proportion of host types with an aggregated distribution. C) $60 \%$ proportion of host types with a fragmented distribution. D) $60 \%$ proportion of host types with a fragmented distribution.

\section{RESULTS}

\subsection{Optimal foraging vs memory with simultaneous seasonality}

Under a simultaneous fruiting scenario, the memory foraging behaviour resulted in relatively low populations in the $60 \%$ proportion with fragmented host distribution (approx. 50\% lower than optimal foraging) and somewhat lower in the $30 \%$ proportion with fragmented hosts. There were similar daily population means in the aggregated simultaneous fruiting scenarios between memory and optimal foraging (fig 3. C). For both foraging strategies under a simultaneous fruiting scenario, relatively more time was spent in good hosts (fig 4.C). For the optimal foraging strategy in the $60 \%$ proportion landscapes, $75 \%$ of the time was spent in good hosts, with an equally low (15-20\%) time spent in poor or average hosts. The same trend was seen in the $30 \%$ proportion landscape, with more time being spent in the non-host as there is more of this cell type available in these landscapes. The memory decay strategy agents spent relatively more time in the poor and average hosts and less time in the good hosts $(<50 \%)$ as compared to the best choice strategy. 

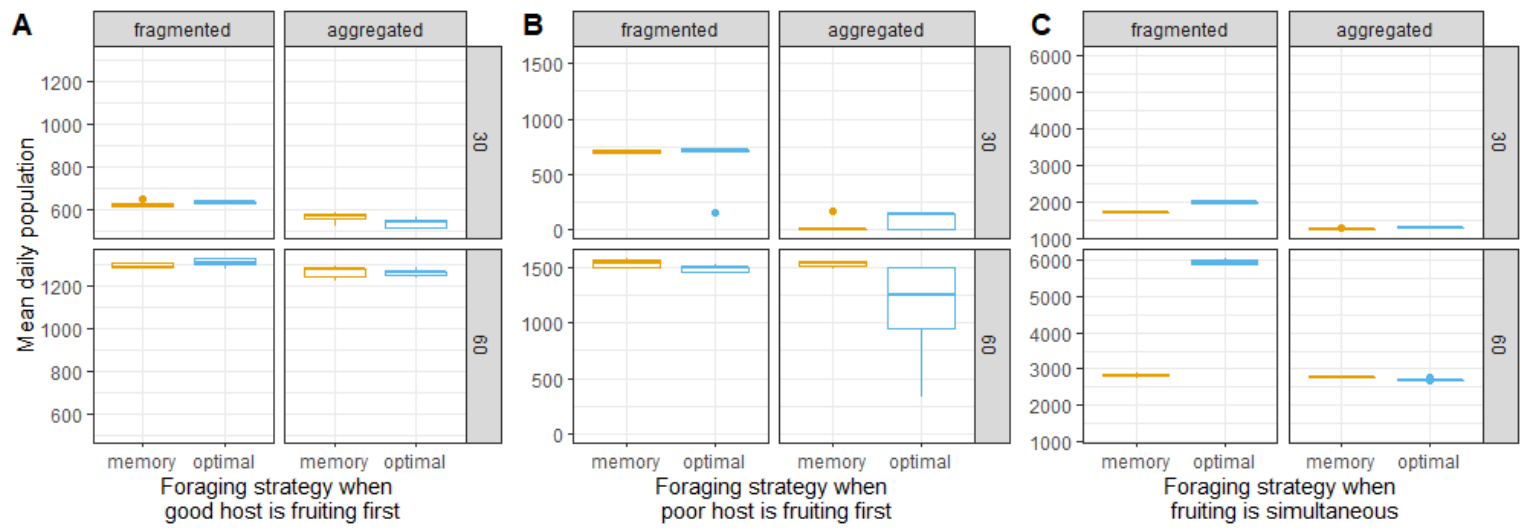

Figure 3. Mean daily population in three fruiting scenarios and four landscapes comparing foraging behaviour with memory and optimal foraging. The four landscapes are combinations of $30 \%$ and $60 \%$ proportion of Bactrocera tryoni hosts, with either fragmented or aggregated distribution. A) Good host fruiting first, B) poor host fruiting first and C) simultaneous fruiting.
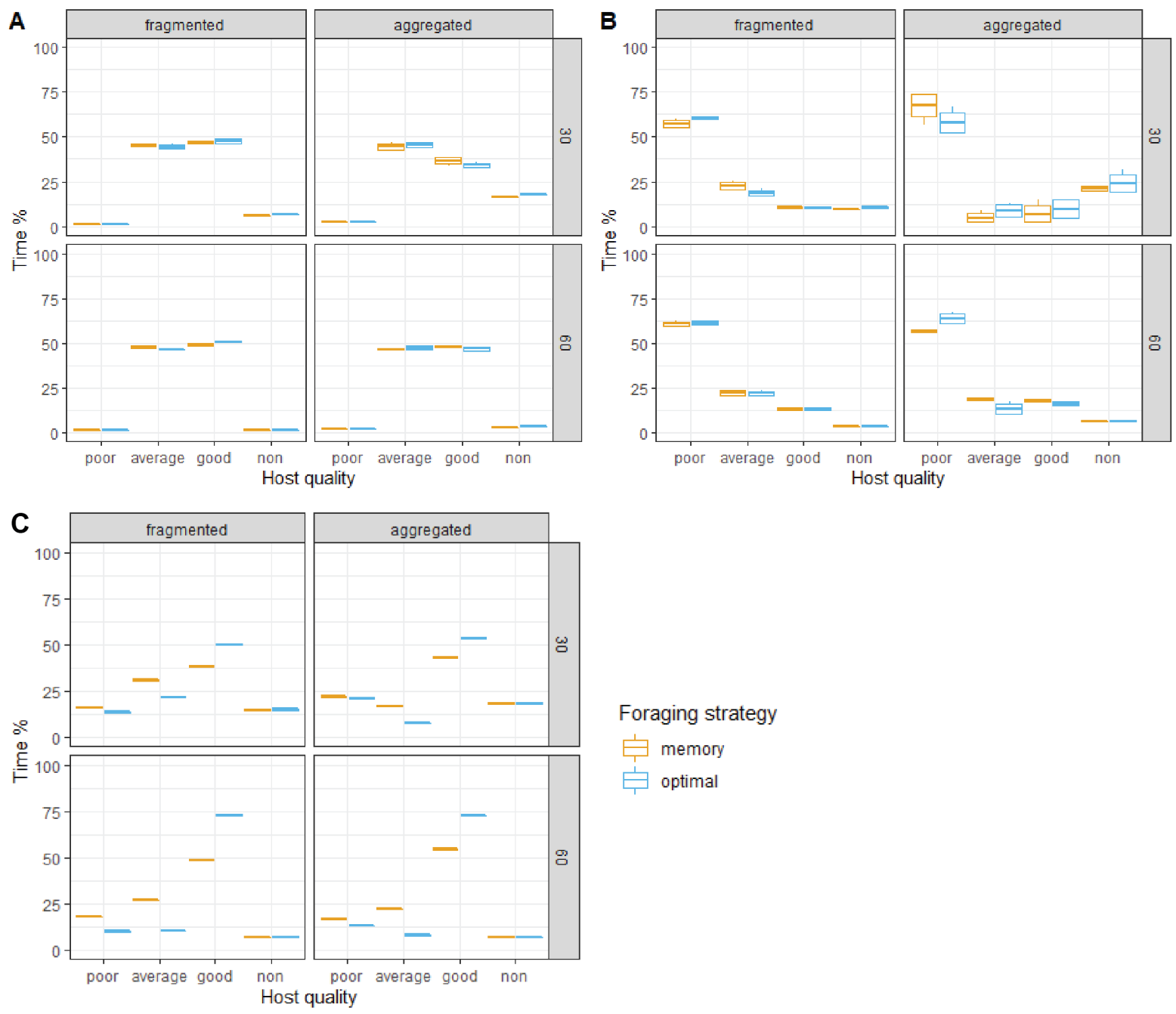

Figure 4. Percentage of time spent in each host time over the simulation for each type of landscape which are described as $30 \%$ and $60 \%$ proportion are host types. The landscape is either aggregated or fragmented. A) Sequential fruiting scenario with the poor host fruiting first. B) Sequential fruiting scenario with the good 
host fruiting first. C) Simultaneously fruiting landscape where all hosts are fruiting at the same time. The behaviour strategies are memory and optimal foraging.

\subsection{Sequential seasonality}

The sequential fruiting scenarios (fig 3. A and B) had similar mean daily population regardless of the foraging behaviour. When the fruit was available sequentially both foraging strategies spent the same amount of time within each fruit type. (fig 4. A and B). The amount of time spent in hosts differed between the sequential fruiting scenarios: when the good host was available first, more time was spent in the good and average hosts and very little time was spent in the poor host. Whereas when the poor host was available first then significantly more time was spent in the poor host compared to the average and good quality hosts. This did not seem to be affected by the foraging strategy, indicating that it is only when fruit is available simultaneously that this strategy may influence population dynamics (depending on the landscape context).

\section{DISCUSSION}

We can use the behavioural data from laboratory studies incorporated into individual-based simulation models to test hypotheses of emergent patterns that could occur due to foraging strategies. Doing a field study of this kind of behaviour would be incredibly difficult and time consuming. By running simulations, we can observe emerging patterns and potentially design experiments or management strategies more efficiently or effectively.

We were expecting a reduction in mean daily population with an aggregated landscape. Instead, the only major difference between the optimal and memory foraging was when host types was simultaneously fruiting and at $60 \%$ host proportion with a fragmented distribution. This result was unexpected and prompts further investigation. What we expected was a "Ground hog day" like pattern where memory foraging behaviour would lead to agents spending more time in the poor host first scenario resulting in a lower population in aggregated environments. It is likely that the carrying capacity is interacting with the model output in a complex manner, since the time spent in the simultaneous scenario in the $60 \%$ proportion does not differ substantially between fragmented and aggregated distribution.

The time spent in each host type gives an insight into the "Ground hog day" scenario especially when simultaneously fruiting. Memory may be detrimental to overall fitness when there are multiple fruits available at the same time. When there is only one fruit available at a time the order of host fruiting is important. In a farming context the timing of fruit availability could be adjusted to reduce population size. More experiments and validation is needed to investigate the role of memory in a local landscape, though it is an interesting first look into the benefits and consequences of foraging behaviour in a polyphagous herbivore insect.

\section{REFERENCES}

Balagawi, S., 2007. Comparative ecology of Bactrocera cucumis (French) and Bactrocera tryoni (Froggatt) (Diptera: Tephritidae) - Understanding the life history consequences of host selection and oviposition behaviour. Griffith University.

Balagawi, S., Vijaysegaran, S., Drew, R.A.I., Raghu, S., 2005. Influence of fruit traits on oviposition preference and offspring performance of Bactrocera tryoni (Froggatt) (Diptera: Tephritidae) on three tomato (Lycopersicon lycopersicum) cultivars. Australian Journal of Entomology 44(2) 97-103.

Becher, M.A., Grimm, V., Knapp, J., Horn, J., Twiston-Davies, G., Osborne, J.L., 2016. BEESCOUT: A model of bee scouting behaviour and a software tool for characterizing nectar/pollen landscapes for BEEHAVE. Ecological Modelling 340 126-133.

Bernays, E.A., 2001. Neural limitations in phytophagous insects: Implications for diet breadth and evolution of host affiliation. Annual Review of Entomology 46(1) 703-727.

Bracis, C., Gurarie, E., Van Moorter, B., Goodwin, R.A., 2015. Memory effects on movement behavior in animal foraging. PLOS ONE 10(8) e0136057.

Dubois, T., Pasquaretta, C., Barron, A.B., Gautrais, J., Lihoreau, M., 2021. A model of resource partitioning between foraging bees based on learning. PLoS Computational Biology 17(7) e1009260.

Gautestad, A.O., 2011. Memory matters: influence from a cognitive map on animal space use. Journal of Theoretical Biology 287 26-36. 
J D Newman et al., Does memory help optimise fruit fly foraging at the local landscape level?

Guttal, V., Romanczuk, P., Simpson, S.J., Sword, G.A., Couzin, I.D., 2012. Cannibalism can drive the evolution of behavioural phase polyphenism in locusts. Ecology Letters 15(10) 1158-1166.

Kaushik, P.K., Renz, M., Olsson, S.B., 2020. Characterizing long-range search behavior in Diptera using complex 3D virtual environments. Proceedings of the National Academy of Sciences 117(22) 1220112207.

Menzel, R., 1999. Memory dynamics in the honeybee. Journal of Comparative Physiology A 185(4) 323-340.

Potting, R.P.J., Perry, J.N., Powell, W., 2005. Insect behavioural ecology and other factors affecting the control efficacy of agro-ecosystem diversification strategies. Ecological Modelling 182(2) 199-216.

RCore, 2020. R: A Language and Environment for Statistical Computing. R Foundation for Statistical Computing: Vienna, Austria.

RStudio, 2016. RStudio: Integrated Development for R. RStudio, Inc: Boston, MA.

Silva, R., Clarke, A.R., 2020a. Does previous experience enhance foraging on a particular host in a polyphagous frugivore? Entomologia Experimentalis et Applicata 168(8) 610-617.

Silva, R., Clarke, A.R., 2020b. The "sequential cues hypothesis": a conceptual model to explain host location and ranking by polyphagous herbivores. Insect Science 27(6) 1136-1147.

Silva, R., Merkel, K., Clarke, A.R., 2020. Memory duration is positively correlated with host fruit preference and offspring performance in a polyphagous frugivore. Austral Entomology.

Stringer, L.D., Kean, J.M., Beggs, J.R., Suckling, D.M., 2017. Management and eradication options for Queensland fruit fly. Population Ecology 59(3) 259-273.

Taillandier, P., Gaudou, B., Grignard, A., Huynh, Q.-N., Marilleau, N., Caillou, P., Philippon, D., Drogoul, A., 2019. Building, composing and experimenting complex spatial models with the GAMA platform. GeoInformatica 23(2) 299-322. 